\section{A review of 145234 ophthalmic patient episodes lost to follow-up}

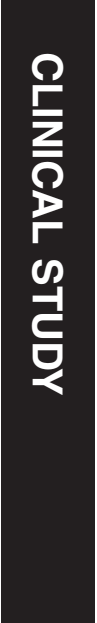

Moorfields Eye Hospital NHS Foundation Trust, London, UK

Correspondence: A Davis, Moorfields Eye Hospital, City Road, London EC1V 2PD, UK

Tel: +44 (0)20 8725 1794; Fax: $+44(0) 2087250372$. E-mail: alison.davis@ moorfields.nhs.uk

Received: 4 February 2016 Accepted in revised form: 13 September 2016 Published online: 11 November 2016

\begin{abstract}
Purpose Lost to follow-up and delays in follow-up care are a major problem in chronic diseases, particularly when irreversible progression precedes symptoms. The NPSA Glaucoma Safety Alert in 2009 highlighted the risk and requirements for consistent robust review systems in ophthalmology. In response, Moorfields Eye Hospital reviewed the records of all patients in all subspecialties without review appointments booked. The purpose of this study was to determine whether ophthalmic patients lost to follow-up had come to harm and develop investigation techniques to optimise safety, which do not put excessive demands on clinical staff time.

Methods The health records of all patients lost to follow-up (LTFU) between July 2007 and November 2012 were reviewed for evidence of clinical harm using a risk-based strategy involving an initial administrative review, then a clinician led electronic patient record review, followed by a review of paper records by clinicians. The final stage was a clinical outpatient review where required determined by clinical risk.

Results Patients identified as lost to follow-up were 145 234; 79562 episodes were closed following administrative review; 50519 were discharged following clinician examination of paper records; 12316 patients required clinical review; and 16 serious incidents were identified, of which 14 patients had glaucoma, 1 a medical retinal condition with secondary glaucoma, and 1 an oculoplastic condition. A number of actions implemented hospital wide are described which minimise future risk.

Conclusion Risk from delays or lost to follow-up care continue and require better capacity and more accurate data nationally. Eye (2017) 31, 422-429; doi:10.1038/eye.2016.225; published online 11 November 2016
\end{abstract}

A Davis, A Baldwin, M Hingorani, A Dwyer and D Flanagan

\section{Introduction}

In 2013/2014, ophthalmology accounted for $8.3 \%$ (6.8 million) of all NHS outpatient episodes. ${ }^{1}$ This workload has markedly and progressively increased in recent years. As the UK's largest specialist eye hospital, Moorfields Eye Hospital (MEH) saw 396058 outpatients in 2010/11, the midpoint of this project, compared with 509,052 in 2014/15. In the two busiest subspecialties, MEH activity increased in glaucoma by 27\% (73 764 in November 2010; 101625 in May 2014) and in medical retina by $36 \%$ (79 226 to 107888 ).

In 2009, the National Patient Safety Agency (NPSA) issued a rapid response report. ${ }^{2}$ Patients with glaucoma were highlighted as being vulnerable to harm following the identification of 44 glaucoma patients who had experienced deterioration of vision, including 13 reports of total loss of vision, due to delayed follow-up appointments from June 2005 until May 2009. As a result of these incidents, the NPSA asked NHS secondary care organisations to review their systems and processes to minimise the risk of avoidable sight loss for patients with established or suspected glaucoma.

As the NPSA alert work was undertaken and awareness increased, Moorfields declared one serious incident (SI) related to a delay in treatment from 2009 to 2011. As part of the action plan to identify and manage ongoing risks for all our patients, at the end of November 2012 $\mathrm{MEH}$ agreed with commissioners to instigate a 5-year look-back review of all patient episodes with unknown outcomes (potential 'lost to follow ups', LTFUs) between July 2007 and November 2012, for all subspecialties. It was not possible to review activity further back due to changes in our patient administrative system (PAS) in 2007.

The methodology and results of that work are presented here to support other ophthalmic 
providers and commissioners faced with managing similar risks.

\section{Subjects and methods}

Patients for whom no outcome was recorded following their appointment, possible LTFU, were identified using the hospital PAS between 1 January 2007 and 30

November 2012 when the project was initiated.

Risk stratification to prioritise the work stream was undertaken to allow those patients at greatest risk of harm to be reviewed early in the project. Factors such as the clinical requirement for early or regularly timed care, risk of irreversible harm, risk of asymptomatic harm and vulnerability of the patient group were taken into account. The review work for glaucoma was already underway at the time of the commencement of the project, and had been previously agreed to be the highest risk area. All patient episodes were reviewed in a stepwise manner and outcomes confirmed. Patients who could not be discharged by an earlier step moved onto the next step.

\section{Step 1: administrative staff}

Patients known to have died were discharged from PAS. Patients who had an electronic patient record letter which indicated that the patient had been discharged from the service were discharged from PAS.

\section{Step 2: clinical staff, review of electronic records}

Senior clinical staff (consultant or senior fellow) reviewed the electronic patient information (partial clinical records and imaging data). If there was sufficient information to make an informed clinical decision the patient was discharged. A letter of explanation was sent to the patient and GP.

\section{Step 3: clinical staff, review of paper records}

Paper notes were then reviewed by the senior clinician for all remaining patients to identify the next actions. Where patients were assessed as not requiring a secondary care appointment, the service wrote to the patient's GP and/or optometrist and the patient advising a community care plan and the patient was discharged.

\section{Step 4}

Where patients required a routine secondary care appointment, this was booked at a clinically appropriate timescale and the patient notified. Where there were patients who required a hospital appointment and there was concern about delay, dedicated clinical sessions were established to review these patients.

\section{Step 5}

Any case identified as having come to harm as a result of a delayed appointment was reported via the Trust incident reporting procedure. If there was the possibility of the patient having come to serious harm the case was discussed at the Serious Incident Panel. If significant harm occurred, this was declared as a serious incident, investigated formally and Duty of Candour requirements followed.

\section{Results}

Patient episodes that were identified as LTFU were 145 234. Table 1 shows the number of episodes by subspecialty service, risk stratification, and the relative subspecialty spilt of Trust activity data during the time period under review.

The largest proportion of episodes were within the general ophthalmology and support services (eg, optometry and orthoptics). These were judged to be low risk. Glaucoma accounted for 3.6\% of the LTFUs and medical retina, deemed the second highest risk area, $10.7 \%$. The proportion of LTFUs was compared with the subspecialty activity, to see whether the LFTU rate was broadly proportional to the amount of activity in each area. The services with more than expected unknown outcomes were general ophthalmology, optometry, support services and orthoptics. Glaucoma, medical retina and external disease had less than expected. Cataract, neuro-ophthalmology, adnexal and paediatrics had slightly less than expected and adult strabismus and vitreoretinal were as expected.

For the percentage LTFU by subspecialty activity during the time period of the project the highest percentage of LTFU by subspecialty activity were again within specialities deemed to be at low risk i.e. support subspecialties (15.8\%), optometry and refraction (14.9\%), and general ophthalmology (11.1\%). Of the subspecialties deemed to be at greatest risk the highest percentage of LTFU was in neurophthalmology $(6.1 \%)$ and paediatrics $(5.2 \%)$. For the other highest risk specialities, glaucoma and medical retina, the percentages were low $(1.3 \%$ and $3.6 \%$, respectively).

Figure 1 shows the progression of the project. Following the completion of step one, 79562 (54.8\%) patient episodes were closed. Following steps two and three, a further 50519 (34.8\%) patients were discharged. These records had clinical evidence that the patient should have been discharged at their last visit or the senior clinician reviewer determined that no further 
Table 1 Number of patient episodes lost to follow-up by service and Trust activity

\begin{tabular}{|c|c|c|c|c|c|c|}
\hline $\begin{array}{l}\text { Risk } \\
\text { Stratification }\end{array}$ & Service & $\begin{array}{c}\text { LTFU Patient } \\
\text { episodes }\end{array}$ & $\begin{array}{l}\% \text { of all } \\
\text { LTFU }\end{array}$ & $\begin{array}{l}\text { Trust activity } \\
2007-2012^{\mathrm{a}}\end{array}$ & $\begin{array}{c}\% \text { Trust activity by } \\
\text { service }\end{array}$ & $\begin{array}{c}\% \text { per service } \\
\text { LTFU }\end{array}$ \\
\hline 1 & Glaucoma & 5251 & 3.62 & 410060 & 17.8 & 1.3 \\
\hline 2 & Medical Retina & 15534 & 10.70 & 431920 & 18.8 & 3.6 \\
\hline 3 & Neurophthalmology & 1206 & 0.83 & 19677 & 0.9 & 6.1 \\
\hline 4 & External Diseases & 6752 & 4.65 & 186748 & 8.1 & 3.6 \\
\hline 5 & Paediatrics & 5418 & 3.73 & 103661 & 4.5 & 5.2 \\
\hline 6 & Strabismus & 2411 & 1.66 & 72065 & 3.1 & 3.3 \\
\hline 7 & Adnexal & 7373 & 5.08 & 97248 & 4.2 & 7.6 \\
\hline 8 & Vitreoretinal & 7688 & 5.29 & 110341 & 4.8 & 7.0 \\
\hline 9 & Anaesthetics & 291 & 0.20 & 3253 & 0.1 & 8.9 \\
\hline 10 & Cataract & 7203 & 4.96 & 156509 & 6.8 & 4.6 \\
\hline 11 & $\begin{array}{l}\text { General } \\
\text { Ophthalmology }\end{array}$ & 31117 & 21.43 & 279858 & 12.2 & 11.1 \\
\hline 12 & Support Services & 18986 & 13.07 & 120128 & 5.2 & 15.8 \\
\hline 14 & $\begin{array}{l}\text { Optometry and } \\
\text { refraction }\end{array}$ & 27168 & 18.71 & 182606 & 7.9 & 14.9 \\
\hline 15 & Orthoptics & 7930 & 5.46 & 101840 & 4.4 & 7.8 \\
\hline 16 & Unknown Service & 0 & 0.00 & 10193 & 0.4 & 0.0 \\
\hline & Total & 145234 & 100.00 & 2298268 & 100 & \\
\hline
\end{tabular}

Abbreviation: LTFU, Lost to follow-up. ${ }^{\text {a }} 1$ April 2007 to 11 Nov 2012.

follow-up was required as the condition was minor or self-limiting. At step 3 it was identified that 15153 (10.4\%) patient episodes required clinical review. For 12316 $(8.5 \%)$ an appointment was made for follow-up in dedicated outpatient clinics (step 4). Sixteen patients were identified as coming to serious harm (step 5) and investigated through the Trust serious incident process.

A total of 2837 (1.95\%) patient episodes could not be closed and work is ongoing to address these episodes. Of these, 682 are episodes with linked appointments with support services and the patients remain under review in an ophthalmic subspecialty but have been discharged from the support service. A total of 1146 were Accident and Emergency patients at one of our sites. The notes are off site and are gradually being reviewed as available. A clinical review of a sample 50 sets of these patient episodes has shown no clinical harm. There are 246 patients in the external disease service for whom paper notes were not initially available as they were stored off site. These notes are now available and are currently being reviewed. For 763 patient episodes no clinical information is available either electronically or on paper. For these patient episodes a letter has been written to the GP and patient offering to arrange a further appointment.

\section{Reasons for lost to follow-up}

Table 2 shows reasons for LTFU. Just over half (79 652; $54.8 \%$ ) of the patient episodes were due to incomplete administrative processes to discharge patients. A total of
18098 (12.5\%) episodes were related to at least one missed booked clinic appointment (a do not attend or 'DNA') and 47574 (32.8\%) were due to cancelled appointments. The PAS records did not contain sufficient information to determine whether this was a hospital- or patient-generated cancellation for all the patient episodes.

For the 16 patients who came to serious harm as a result of being lost to follow-up (Table 3) a more detailed analysis was undertaken. This analysis showed that the reasons for LTFU were multifactorial. The serious incidents were distributed evenly across the years of the project. There were 14 patients with glaucoma, one with a central retinal vein occlusion (CRVO) and secondary glaucoma following a dexamethasone intravitreal implant and one with a benign pleomorphic adenoma of the orbit. For glaucoma patients, 10/14 (72\%) were the result of no follow-up appointments being made after an outpatient visit. In addition, one patient had been seen in $A \& E$, referred to the glaucoma service but no glaucoma appointment was made; one patient DNA'd and was then not rebooked; one patient had no follow-up appointment made after laser trabeculoplasty; one patient did not receive an appointment after the initial GP referral, was subsequently seen and then LTFU after the outpatient appointment.

There was recorded evidence in the notes suggesting a degree of vulnerability for some of the glaucoma patients (one had dementia, one had a poor memory due to a brain haemorrhage, two had documented poor compliance with therapy, and one attended multiple appointments 


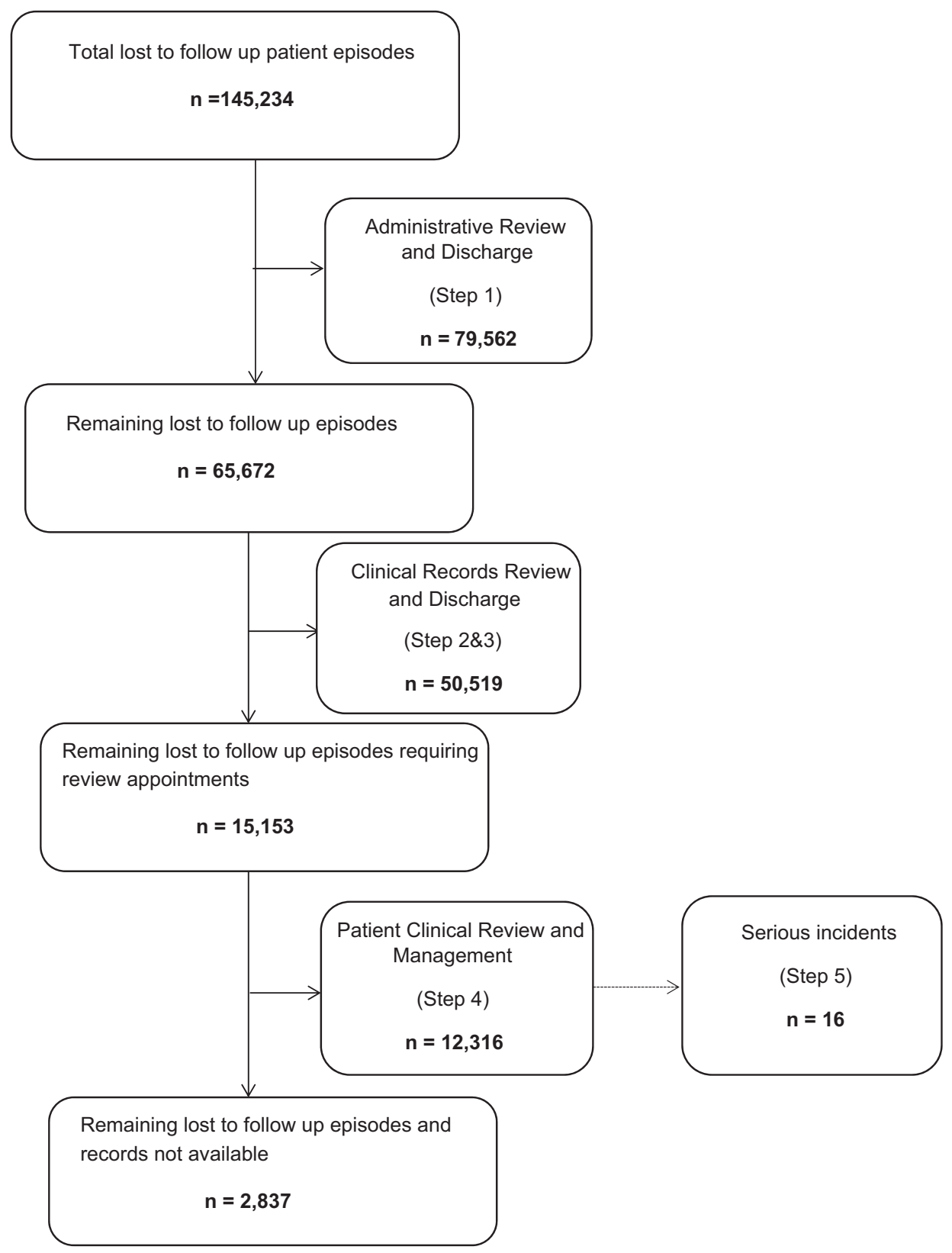

Figure 1 Results of lost to follow-up.

for diabetic retinal screening in addition to his glaucoma appointments). All the glaucoma patients had significant visual field loss as a result of being LTFU.

For the patient with a CRVO no appointment was made for review after his dexamethasone implant. 12 months later he re-presented with secondary glaucoma. One adnexal patient without harm was classified as an SI due to the seriousness of potential harm, that is, a 'near miss'. The patient had undertaken the required treatment outside the UK without notifying the hospital and then returned to $\mathrm{MEH}$ post treatment.

\section{Discussion}

This project was initiated as a consequence of our action plan for the NPSA alert for glaucoma ${ }^{2}$ and a serious incident relating to a glaucoma patient in $\mathrm{MEH}$. The key point of the NPSA alert was to ensure that all patients with ocular hypertension, suspected, or diagnosed glaucoma were monitored within the safe intervals defined in the NICE glaucoma guidance. ${ }^{3,4}$ It became clear, as we reviewed our glaucoma patients that there were significant numbers of patients in other subspecialties for whom we had no recorded outcome 
Table 2 Reasons for lost to follow-up

\begin{tabular}{lcc}
\hline Reason & Number & $\%$ \\
\hline Incomplete administrative processes & 79562 & 54.8 \\
DNA & 18098 & 12.5 \\
Cancellations & 47574 & 32.7 \\
Total & 145234 & 100 \\
\hline
\end{tabular}

following their last clinic visit. We therefore proactively extended this project beyond glaucoma to prevent harm to ophthalmic patients across all ophthalmic subspecialties. Comparing the LTFU rate with the proportion of subspecialty activity, fewer glaucoma patients than expected had unknown outcomes. The data for LTFU by percentage of subspecialty activity also confirm a small percentage of LTFU for glaucoma. We suspect that this is because the work to reduce the risk for glaucoma patients with unknown outcomes preceded the onset of our formal study. It is possible, although less likely, that we have not identified all glaucoma patients who have been LTFU, as the data for other subspecialties are mainly in line with what would be expected from clinical activity. Medical retina, despite being a speciality known to have a large follow-up workload, had a smaller percentage of LTFU than the more surgically biased subspecialties. This would suggest that with due diligence it is possible to manage LTFU to reduce risk to patients. The percentage LTFU for neurophthalmology and paediatrics was high, which was of concern due to the nature of the clinical conditions and safeguarding issues.

There has been concern since the NSPA alert that there is still a significant national risk of permanent harm due to LTFUs or delays in follow-up clinic appointments from a significant mismatch between ophthalmic capacity and demand. Bodies such as the Royal College of Ophthalmologists, the RNIB and the Macular Society have raised concerns from 2012 onwards. ${ }^{5-7}$ Most recently, in the 2015, Addenbrookes Hospital (Cambridge University Hospitals NHS Foundation Trust) CQC report, significant and repeated mention is made of unsafe delays to ophthalmic follow-up in clinics causing serious incidents and significant harm. ${ }^{8}$

The Royal College of Ophthalmologists has an ongoing British Ophthalmic Surveillance Unit study to ascertain the number of patients nationally who have experienced deterioration of sight caused by delay in review or treatment. The initial study findings consistently report 20 cases per month of whom $42 \%$ have glaucoma, $20 \%$ diabetic retinopathy, 6\% AMD, and 32\% with other diagnoses (B Foot. Personal Communication).

In a previous study of glaucoma patients from one site within our institution, $8 \%$ had disease progression that might have been prevented if there been adherence to the clinically planned follow-up regime. ${ }^{9}$ In our project, we identified 16 patients who had come to serious harm as a result of LTFU. Although this is a small number in the overall context of the project, for all of these patients it had a profound effect on their vision, which could have been prevented. Nearly all $(88 \%)$ of the patients identified within our project who came to serious harm had glaucoma. All of these patients had significant visual field loss, and in five patients the visual field was extinguished in one or both eyes. It is likely that glaucoma patients are at the highest risk of harm from LTFU because glaucoma is often asymptomatic until end stage disease leads to significant visual field and vision loss.

\section{What are the causes are of LTFU?}

Failure of patients to attend clinics is a known and significant problem. Previous studies have shown that the reasons vary by age, gender, ethnicity, socioeconomic status and medical speciality.

A study in 1994 from another specialist eye centre found that, of patients who DNA, $27.3 \%$ were due to clerical errors and $17.9 \%$ were due to the patient forgetting an appointment. They suggested that telephone reminders and better access to the correct department could reduce the number of non-attendances by up to $40 \% .{ }^{10}$ A similar study in a neurology outpatient clinic found that $28 \%$ of patients who failed to attend an appointment simply forgot. ${ }^{11}$ In another study, to identify why patients might miss an appointment, the main risk factors were found to be older age and non-English proficiency. ${ }^{12}$ A study of the views of health-care professionals as to why children did not attend a general paediatric appointment also suggested the reasons were multifactorial ${ }^{13}$ and included socioeconomic factors, issues relating to other children as well as factors relating to hospital administration. King et al, ${ }^{14}$ found a $12.9 \%$ DNA rate in a study in a specialist eye hospital in 1991 but did not find any specific reasons for patients failing to attend.

In our project, the factors identified as contributing to patients being LTFU, whether or not there was harm, were multiple but commonly included patient DNA, hospital cancellations or rearrangements of clinic dates, frequently worsened by capacity limitations and difficulties getting through on the telephone. In addition, capacity issues meant that clinics often finished late so that the receptionists had left and were not available to collect outcome forms or book the next appointment for the patient. We do not have data for the full project of the number of cancellations. However, data from the first 99659 patient episodes reviewed were that there were 13974 cancellations with 4909 (5\%) 
Table 3 Serious incidents

\begin{tabular}{|c|c|c|c|c|c|c|}
\hline Patient & Diagnosis & Reason for lost to follow-up & Age & $\begin{array}{l}\text { Duration of } \\
\text { lost to follow- } \\
\text { up (months) }\end{array}$ & $\begin{array}{l}\text { Significant } \\
\text { fields loss }\end{array}$ & Comments \\
\hline 1 & POAG & No follow-up appointment booked & 44 & 25 & Yes & Poor compliance \\
\hline 2 & $\begin{array}{l}\text { Left orbital } \\
\text { pleomorphic } \\
\text { adenoma }\end{array}$ & $\begin{array}{l}\text { Notes lost and No follow-up appointment } \\
\text { booked }\end{array}$ & 24 & 4 & No & No clinical harm \\
\hline 3 & $\begin{array}{l}\text { Glaucoma } \\
\text { secondary to } \\
\text { steroid response }\end{array}$ & No follow-up appointment booked & 74 & 12 & Yes & \\
\hline 4 & POAG & No follow-up booked & 60 & 72 & Yes & $\begin{array}{l}\text { Seen in another ophthalmic } \\
\text { service }\end{array}$ \\
\hline 5 & POAG & No follow-up booked after being seen in $\mathrm{A} \& \mathrm{E}$ & 66 & 7 & Yes & \\
\hline 6 & POAG & No follow-up appointment booked & 66 & 50 & Yes & Poor English \\
\hline 7 & POAG & No follow-up appointment booked & 59 & 16 & Yes & \\
\hline 8 & $\begin{array}{l}\text { Low tension } \\
\text { glaucoma }\end{array}$ & No laser trabeculoplasty booked & 83 & 19 & Yes & \\
\hline 9 & POAG & $\begin{array}{l}\text { No action following DNA, some evidence } \\
\text { seen at another hospital during this time, but } \\
\text { no regular appointments }\end{array}$ & 85 & 81 & Yes & Dementia \\
\hline 10 & POAG & No follow-up appointment booked & 72 & 47 & Yes & \\
\hline 11 & POAG & No follow-up booked & 66 & 45 & Yes & $\begin{array}{l}\text { Poor compliance, memory } \\
\text { loss secondary to brain } \\
\text { haemorrhage, NIDDM }\end{array}$ \\
\hline 12 & $\begin{array}{l}\text { Glaucoma } \\
\text { secondary to } \\
\text { intravitreal } \\
\text { dexamethasone }\end{array}$ & $\begin{array}{l}\text { No follow-up booked after intravitreal } \\
\text { dexamethasone injection }\end{array}$ & 40 & 12 & Yes & Also had CRVO \\
\hline 13 & POAG & No follow-up booked & 47 & 55 & Yes & \\
\hline 14 & POAG & No follow-up appointment booked & 73 & 65 & Yes & $\begin{array}{l}\text { Continued treatment } \\
\text { despite no follow-up }\end{array}$ \\
\hline 15 & $\begin{array}{l}\text { Acute Angle } \\
\text { Closure Glaucoma }\end{array}$ & No follow-up booked & 80 & 16 & Yes & \\
\hline 16 & Advanced POAG & $\begin{array}{l}\text { Follow-up appointment cancelled by } \\
\text { hospital, patient then DNA'd appointment, } \\
\text { patient discharged, represented to A\&E }\end{array}$ & 52 & 81 & Yes & $\begin{array}{l}\text { Ambulance driver, no } \\
\text { longer able to drive }\end{array}$ \\
\hline
\end{tabular}

Abbreviations: A\&E, accident and emergency; DNA, did not attend; CRVO, central retinal vein occlusion; NIDDM, non-insulin-dependent diabetes mellitus; POAG, primary open-angle glaucoma.

due to the hospital and 9065 (9\%) being patient-related cancellations.

The detailed investigations from our patients who came to serious harm from being LTFU found that $75 \%$ of patients had no appointment booked although follow-up was clearly planned by a clinician. In all of these cases there was at least some element of appointments being changed usually, but not always, by the hospital. Other contributing issues were lost notes, failure to book a procedure or the transferring of care from one subspecialty area to another particularly via paper referrals.

Protecting vulnerable adults is an obligation for all NHS providers. ${ }^{15}$ Two of our patients who came to serious harm were vulnerable adults. For these patients vulnerability due to age, mental infirmity, and not speaking English as a first language contributed significantly.

\section{What measures can be taken to reduce LTFUs?}

Orthopaedics has a similar outpatient workload to ophthalmology. ${ }^{1}$ Schoh et al ${ }^{16}$ found that a multipronged approach was successful, including combination of waiting list audits, triage guidelines, non-medical (physiotherapy) led clinics, a clear DNA policy, a specialist lead nurse role and a patient-focussed booking system, and resulted in a $66 \%$ reduction in the number of patients waiting for their first appointment, an $87 \%$ reduction in the waiting time from referral to first appointment and a $10 \%$ reduction in new patient DNA rate. 
A Cochrane systematic review ${ }^{17}$ of the use of SMS text messaging reminders in a number of countries found that such reminders have a moderate effect in improving attendance figures.

We have developed our processes as follows:

1. Administrative support: administrative staff have been trained as to the importance of ensuring each patient has an outcome at the end of clinic and it is Trust policy that receptionists remain until the last patient has left.

2. Clinical input: case notes for patients who do not attend or whose appointment is cancelled by either the hospital or the patient are reviewed by a senior clinician. Discharge or follow-up is arranged as clinically appropriate.

3. Access policy: This has been rewritten with an emphasis on ensuring that all patient episodes have a recorded outcome at the end of clinic. The Trust now very actively manages DNAs so that, where possible and safe, patients are discharged back to their GP and all parties informed clearly of this action and its implications.

4. Clear identification and monitoring: the Trust keeps a $\log$ of non-outcomed patients for each site and service as part of the weekly administration pack which is actively monitored by senior clinicians and managers. This report is discussed at local meetings and at the Trust Clinical Governance Committee.

5. Text and telephone: the Trust has a text reminder system for all patient 2 days before their appointment. We have piloted phoning patients 2 days in advance to increase the likelihood of them attending. Initial work has shown this to be highly successful.

6. Raising awareness: there have been numerous presentations in business, governance and teaching meetings regarding this issue, the potential for harm and the requirement for all staff to take action to protect patient safety and ensure robust booking arrangements with senior clinical input.

7. Internal referrals: there is further work to be done to ensure that patients referred internally to another ophthalmic subspecialty services have the appropriate appointment made.

We have had no further serious incidents as a result of LTFU since the launch of this project but it is important to note the expected number of serious incidents as a result of LTFU is small for any one unit and preventing poor patient outcomes as a result of patients being LTFU requires constant vigilance.

\section{Conclusions}

This paper describes our experience of completing an exercise to resolve LTFUs in a specialist eye hospital. We describe the methodology developed to conduct such a review across a large population of patients. These principles can be applied to other ophthalmic hospitals, hospital ophthalmology departments, and outside ophthalmology. A plan for managing LTFUs is particularly important for management of chronic disease and for services with a high proportion of vulnerable patients. This study has confirmed the suspicion that glaucoma patients are the most vulnerable patient group.

Nationally, a key preventative strategy for this will be addressing the risk for LFTU or outpatient delay presented by the significant lack of capacity for ophthalmic care. RTT18 rules require that patients are given follow-up appointments at or near to the planned time for clinical review. ${ }^{18}$ In our view, national consideration should be given to create targets for reporting how well providers are managing follow-up patients.

\section{Summary}

\section{What was known before}

- Ophthalmology is one of the largest providers of outpatient care in the NHS.

- Glaucoma patients are vulnerable if outpatient follow-up appointments are delayed.

What this study adds

- Using a structured methodology it is possible to review a large number of patients lost to follow-up efficiently and effectively.

- Using this methodology all patients at risk of serious harm if lost to follow-up can be identified.

\section{Conflict of interest}

The authors declare no conflict of interest.

\section{References}

1 Hospital Episode Statistics. Hospital Outpatient Activity 2013-2014. Available at http://www.hscic.gov.uk/ catalogue/PUB16722/hosp-outp-acti-2013-14-summ-reporep.pdf.

2 National Patient Safety Agency Preventing delay to followup for patients with glaucoma. Available at Rapid Response Report 2009; NPSA/2009/RRR004.

3 Department of Health. NICE glaucoma quality standard, March 2011. Available at http://www.nice.org.uk/ guidance/ qualitystandards/quality standards.jsp. 
4 National Institute of Clinical Excellence (NICE) guidance CG85. Glaucoma: diagnosis and management of chronic open angle glaucoma and ocular hypertension, April 2009. Available at http://guidance.nice.org.uk/CG85.

5 Royal College of Ophthalmologists. 2014 New to follow up (N: F) ratios in ophthalmology outpatient services. Available at https://www.rcophth.ac.uk/wp-content/uploads / 2014/12/2011_PROF_114_New-to-follow-up-ratios-inophthalmology-outpatient-services.pdf.

6 RNIB campaign report. Saving money, losing sight. November 2013. Available at http://www.rnib.org.uk/ campaigning-policy-and-reports-hub-eye-health/eye-healthreports.

7 Royal College of Ophthalmologists and the Macular Society AMD Services Survey Report. 2013. Available at https:// www.rcophth.ac.uk/wp-content/uploads/2015/02/JointRCOphth-and-MS-Survey-Results.pdf.

8 Care Quality Commission Cambridge University Hospitals NHS Foundation Trust, Addenbrooke's and the Rosie Hospitals Quality Report 2015. Available at http://www. cqc.org.uk/location/RGT01/reports.

9 Tatham A, Murdoch I. The effect of appointment rescheduling on monitoring interval and patient attendance in the glaucoma outpatient clinic. Eye 2012; 26: 729-733.

10 Potamitis T, Chell PB, Jones HS, Murray PI. Non-attendance at ophthalmology outpatient clinics. J R Soc Med 1994; 87: 591-593.

11 Roberts K, Callanan I, Tubridy N. Failure to attend outpatient clinics: is it in our DNA? Int J Health Care Qual Assur 2011; 24(5): 406-412.
12 Torres O, Rothberg MB, Garb J, Ogunneye O, Onyema J, Higgins T. Risk factor model to predict a missed clinic appointment in an urban, academic, and underserved Setting. Popul Health Manag 2015; 18(2): 131-136.

13 Cameron E, Heath G, Redwood S, Greenfield S, Cummins C, Kelly D et al. Health care professionals' views of paediatric outpatient non-attendance: implications for general practice. Fam Pract 2014; Vol. 31(1): 111-117.

14 King A, David Jones H, O'Brien C. Factors affecting nonattendance in an ophthalmic outpatient department. J $R$ Soc Med 1995; 8S: 88.

15 Safeguarding Vulnerable People in the Reformed NHS. Accountability and Assurance Framework, NHS Commissioning Board 21 March 2013. Available at http:// www.england.nhs.uk/wp-content/uploads/2013/03/ safeguarding-vulnerable-people.pdf.

16 Schoch PA, Adair L, Schoch PA, Adair L. Successfully reforming orthopaedic outpatients. Aust Health Rev 2012; 36 (2): 233-237.

17 Gurol-Urganci I, de Jongh T, Vodopivec-Jamsek V, Atun R, Car J. Mobile phone messaging reminders for attendance at healthcare appointments. Cochrane Database Syst Rev 2013; 5(12): CD007458.

18 Recording and reporting referral to treatment (RTT) waiting times for consultant-led elective care. Available at https:/ / www.england.nhs.uk/statistics/wp-content/uploads/ sites/2/2013/04/Recording-and-reporting-RTT-guidancev24-2-PDF-703K.pdf. 